\title{
Retroactive interference as a function of degree of interpolated study without overt retrieval practice
}

\author{
DENNIS J. DELPRATO \\ Eastern Michigan University, Ypsilanti, Michigan
}

\begin{abstract}
In two experiments, retroactive interference (RI) was examined under conditions designed to minimize retrieval-induced inhibition and output interference. In Experiment 1, the participants first learned a list of 10 person-location pairs, after which they viewed an interpolated list with the same person terms and different locations for one, four, or eight cycles. Learning was via a study-only method, so that the experimental participants did not perform instructed retrieval of the second list before a cued recall test that asked for List 1 locations only. The experimental participants in Experiment 2 received incidental learning instructions and then viewed a list of 20 unrelated words followed by another list presented either one or four times; the first two letters of each List 1 word were provided on the critical recall test. In both experiments, RI occurred and increased with degree of interpolation. The results suggest that RI does not require retrieval-induced inhibition.
\end{abstract}

Memory researchers have long assumed that retroactive interference (RI) results from interpolated learning and is a negatively accelerated increasing function of the degree of interpolated learning (e.g., Barnes \& Underwood, 1959; Briggs, 1957; Postman, 1971). Classic experimenters usually operationalized degree of interpolated learning in multitrial experiments by varying the number of study and test trials. In most studies, AB-AC paired associates (same cues and different targets across two lists) have been used, but the function has been obtained for serial lists (e.g., McGeoch, 1932; Melton \& Irwin, 1940; Postman \& Riley, 1959), free recall (Postman \& Keppel, 1967), and connected discourse (Slamecka, 1960). Modelers often have cited Barnes and Underwood's study as a classic and have used the data from this experiment to test their models' fit to a fundamental empirical relation (e.g., Chappell \& Humphreys, 1994; Lewandowsky, 1991; McCloskey \& Cohen, 1989; Mensink \& Raaijmakers, 1988).

In view of our longstanding confidence that RI is produced by interfering interpolated learning and that increases in degree of interpolation are accompanied by increases in RI, the outcome of Bäuml's (1996) revisit to this question may appear surprising. Bäuml used a singlepresentation free recall method with lists of 25 words and manipulated degree of interpolated learning by varying the duration of exposure of individual list items. Experiment 2 reported data for first-list (original learning) recall for a control condition and two experimental condi-

This research was supported in part by a grant from the Eastern Michigan University Graduate School Research Support Fund. I thank Veronica Jamieson and Melissa Shue for their help in collecting data. Correspondence concerning this article should be addressed to D. J. Delprato, Department of Psychology, Eastern Michigan University, Ypsilanti, MI 48197 (e-mail: ddelprato@emich.edu). tions. Participants in each experimental condition studied four different interpolated lists. Exposure duration per item in the experimental groups was either 2 or $5 \mathrm{sec}$. Bäuml and subsequent commentators (Anderson, E. L. Bjork, \& R. A. Bjork, 2000) focused on the statistically equivalent first-list recall scores from the two experimental conditions $(54.0 \%$ and $52.4 \%$ for the shorter and the longer exposure durations, respectively) and concluded that the results conflicted with those from earlier studies - degree of interpolated learning did not affect amount of RI. According to Bäuml and Anderson et al. (2000), the new finding is especially noteworthy when one compares Bäuml's methods with those of the classical studies. Whereas most of the previous experiments required learners to repeatedly study and recall (retrieve) items during the course of interpolated learning, Bäuml did not program recall periods during learning.

Bäuml's (1996) failure to obtain differences in RI when degree of interpolated learning increased with increased study time appears to be consistent with Anderson, R. A. Bjork, and E. L. Bjork's (1994) proposal that the classic function relating interpolated learning to RI is attributable to retrieval-induced inhibitory control processes established during interpolated learning, rather than to increased strength of interfering responses and associations. Anderson et al. (1994) pointed out that methods involving study and recall episodes, such as anticipation with paired associates, confound repeated exposure to items (study) with repeated recall attempts (retrieval practice). A substantial body of research indicates that retrieval practice is an independent source of forgetting, perhaps because it activates control processes that contribute to repression-like inhibition of competitors with targets (e.g., Anderson, 2003; Anderson et al., 2000; Anderson et al., 1994). According to this thinking, by providing only study periods without recall episodes, Bäuml 
eliminated forgetting that otherwise would have resulted from retrieval practice and, thereby, eliminated RI and the classic degree-of-interpolated-learning effect.

Bäuml's (1996) data may very well point the way to a new outlook on RI. Certainly, the unlearning hypothesis of interference theory no longer appears capable of contributing to a satisfactory account (Postman, 1976). As was mentioned above, Bäuml did not obtain RI if one compares the recall scores of the longer exposure duration experimental group with those for the shorter duration condition. However, Bäuml's data do reveal RI when the experimental groups' recall scores are compared with those of a conventional RI control - that is, two identically treated groups studied only the first list and yielded a combined mean percentage of recall of 78.6. Thus, from the standpoint of the orthodox definition of RI (e.g., Postman, 1971), Bäuml's findings do not demonstrate absence of RI under conditions of interpolation with no retrieval practice. But two shortcomings of Bäuml's RI control condition temper conclusions from this experiment. The participants were given the final recall test with no delay, after first-list study, to control for the time required to present the interpolated lists to the experimental participants. Thus, control recall scores may be overestimates, in which case the experiment would have led to an overestimation of the amount of RI. However, in the absence of a control for retention interval per se, we have no way of knowing whether the study did overestimate the amount of RI. Bäuml's control data were also problematic because they were obtained from a separate experiment. Principles of experimental control suggest that cross-experiment comparisons, at best, provide preliminary indications of effects that require follow-up with more tightly controlled manipulations.

Anderson (2003) made a strong case that it is not interference and new learning per se that underlie RI. Instead, new learning can, but does not inevitably, establish conditions for the fundamental mechanism that produces the forgetting obtained in RI experiments-namely, inhibition. New learning contributes to forgetting only when it requires the participant to cope with interference (resolve competition) in order to function effectively. Thus, retrieving interpolated targets that are subject to interference from originally learned targets arouses inhibition of the interfering traces (targets). Although, Anderson's theory has been well supported by research in which a preparation devised to test various implications of the construct of retrieval-induced inhibition has been used, Bäuml's (1996) is the only research to date that has used more conventional RI procedures to determine whether RI is eliminated when new learning does not require participants to resolve competition. In the present research, RI was further investigated under conditions designed to minimize retrieval-induced inhibition. In each of two experiments, RI was estimated using a standard rest control condition and more than one level of degree of interpolated study.

\section{EXPERIMENT 1}

Experimental participants studied a list of $A B$ paired associates. After they had been tested for acquisition, different groups studied an interpolated list (IL) of AC pairs for one, four, or eight cycles in the absence of overt retrieval practice. Because increased exposure to interpolated material leads to increased recall of interfering material, there would be more opportunities for output interference of first-list items as degree of interpolated study increased (Smith, 1971). To minimize output interference, testing followed Bäuml's (1996) procedure of not requesting List 2 items until after the participants had recalled List 1.

\section{Method}

Participants and Design. Ninety-seven students participated as part of the requirements for an introductory psychology lab class that consisted of eight sections. They were tested in intact groups with sections randomly assigned to one of four independent conditions, with the restriction that group $n \mathrm{~s}$ be as equivalent as possible. Two sections that met at different times formed each of the four levels of the degree-of-interpolated-learning variable that was manipulated by way of the number of cycles the IL was presented: zero (control), one, four, or eight. The $n$ s for these four conditions were $22,25,24$, and 26 , respectively.

Materials. The materials consisted of lists of 10 person-location pairs (e.g., teacher-airport, artist-church). A single set of persons and a single set of locations were used as the first list for all the groups. Two different random pairings were assigned to the two different lab sections that make up each condition. Another set of person-location pairs with an $\mathrm{AB}-\mathrm{AC}$ relation to the first list served as the IL. A single random pairing of the AC pairs was used as the IL for all the experimental groups.

Procedure. The materials were presented on a computer screen, and booklets were provided for recall tests. The learning instructions encouraged the participants to think of all pairs in terms of the sentence, "The [person] is in the [location]." The 10 pairs of the first list appeared for $4 \mathrm{sec}$, each in a different random order on four cycles. After the last pair, the participants were asked to turn to the second page of the booklet, where they saw the 10 cues in a random order. The instructions were to write as many of the locations as they could next to the person. After a maximum of $3 \mathrm{~min}$ (the same period allowed for all the recall tests), the experimental participants were informed that they would see a second list in which each person from the first list was paired with a different location. The IL was presented at a rate of $4 \mathrm{sec}$ per pair for one (IL-1), four (IL-4), or eight (IL-8) cycles over independent groups. Instead of receiving a second list, the groups assigned to the zero-cycle control condition (IL-0) spent a 6-min interval reading instructions and subsequently rating cartoons that contained no words. After the second list or the cartoon-rating task, all the participants received a cued recall test structured like the one used to assess first-list learning. The instructions for the experimental groups stressed that the test was to determine how well they remembered where the people were originally in the first list. A single blank line was placed to the right of each cue, and blank lines at the bottom of the page were used when the participant could recall a target but was unwilling to guess as to its associated cue. The instructions for a second recall test requested first-list targets and, for the experimental participants, second-list targets.

\section{Results}

Recall protocols were scored in terms of targets correctly placed with cues and targets recalled, irrespective 
of their placement. The two scoring methods yielded comparable conclusions; thus, only the results from the former will be reported. The groups were adequately equated before differential treatment. The mean recall scores (as percentages, standard deviations in parentheses) after the four cycles of first-list study for the four groups were IL- $0=85.9 \%$ (16.2), IL-1 $=87.2 \%$ (16.2), IL-4 $=88.8 \%(14.5)$, and IL-8 $=90.8 \%(13.5)$ $\left[F(3,93)=0.46, M S_{\mathrm{e}}=228.85, p=.71\right]$.

Because RI experiments typically yield performance scores before participants are exposed to experimental manipulations, this type of study provides a built-in covariate that researchers are advised to incorporate into covariance analyses to increase statistical power, a major factor in statistical conclusion validity (Shadish, Cook, $\&$ Campbell, 2002). Power is especially important in cases involving the issue that prompted the present work: the possibility of a lack of covariation between degree of interpolated learning and first-list recall scores. Accordingly, analyses of covariance were used to assess firstand second-list recall on the critical tests. Recall scores after first-list study were used as the covariate for both analyses.

The degree-of-interpolated-learning manipulation had the expected effect. Even though not measured until the second recall test (which could have been biased against memory for the second list), scores increased as a function of degree of exposure of List 2 pairs $[F(2,71)=$ 9.86, $\left.M S_{\mathrm{e}}=534.11, p<.001\right]$. The unadjusted mean percentages of recall (standard deviations in parentheses) for the three groups were IL-1 $=46.8 \%$ (27.6), IL$4=67.1 \%$ (27.1), and IL-8 $=78.5 \%$ (24.8). The adjusted means (based on the analysis of covariance) for the three conditions were $48.3 \%, 67.2 \%$, and $76.8 \%$ for IL-1, IL-4, and IL-8, respectively.

The recall scores on the test that followed interpolated learning or control activity reveal decreases in performance over the four levels of the degree-of-interpolatedlearning variable. Unadjusted mean percentages recall (standard deviations in parentheses) for the four groups were IL-0 = 84.1\% (21.3), IL-1 = 78.0\% (27.2), IL-4 = $70.0 \%$ (28.3), and IL-8 $=63.5 \%$ (28.0). The adjusted means for the four conditions were $87.0 \%, 79.3 \%$, $69.4 \%$, and $60.3 \%$ for IL-0, IL-1, IL-4, and IL-8, respectively. The variation among these means was statistically significant $\left[F(3,92)=9.19, M S_{\mathrm{e}}=349.75, p<\right.$ $.001]$, and they are presented in Figure 1. The Bonferroni method was used to adjust for multiple comparisons (with alpha set at .05). Only the IL-4 and IL-8 groups differed from the IL-0 control, indicating that RI was obtained with 16 and $32 \mathrm{sec}$ of exposure to individual interpolated items, but not with only $4 \mathrm{sec}$ of study per AC pair. The IL-1 versus IL-4 comparison was not significant, but increasing degree of IL from 4 to $32 \mathrm{sec}$ of study per pair increased RI (IL-1 vs. IL-8 comparison). The difference between the IL- 4 and the IL- 8 conditions was not significant.

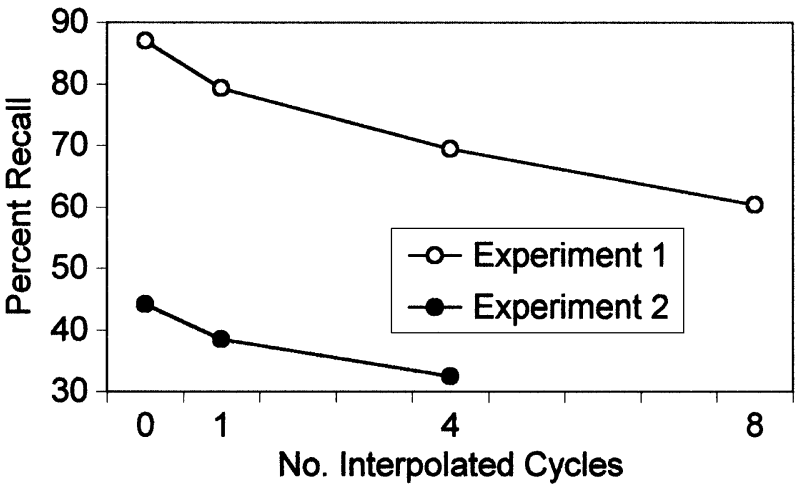

Figure 1. Percentages of recall as a function of number of interpolated list cycles for two experiments (adjusted means shown for Experiment 1).

\section{Discussion}

The main finding of this experiment was that RI occurred and gradually developed over four successive levels of degree of interpolated study in the absence of overt retrieval practice for the interfering list. The finding of $\mathrm{RI}$ is important because Bäuml's (1996) failure to obtain variations in first-list recall over two levels of interpolated learning might suggest that RI is eliminated in the absence of retrieval practice for items that share retrieval cues (e.g., Anderson, 2003). Given the present results, it seems likely that Bäuml's data reflect RI that reached asymptote at the lowest level of the two degrees of IL used in the experiment. RI seems to be more influenced by continued increases in degree of interfering learning than is proactive interference (Underwood, 1983); however, it has not been uncommon for RI to reach a plateau after a relatively low level of interpolation (e.g., McGeoch, 1932; Melton \& Irwin, 1940; Postman \& Riley, 1959; Underwood \& Lund, 1981).

Although the present study yielded RI in the absence of overt retrieval practice, its theoretical implications must be qualified. With the purpose of minimizing output interference, the critical recall test followed Bäuml (1996) by requesting that the participants recall only List 1 targets to the cues. However, if on such a test participants can remember both targets yet are uncertain as to the list of origin of each (a form of list differentiation failure or source confusion), their decision to report the target from List 2 could erroneously reflect List 1 loss. Analysis of List 2 overt intrusions in the three degree-ofIL groups somewhat counters this possibility. Recall protocols were scored for combined specific (List 2 target written next to its corresponding List 1 cue) and general (List 2 target written next to a List 1 cue other than the cue it was paired with) intrusions. In the IL-1 condition, 6 out of 25 participants $(24.0 \%)$ produced a total of 7 intrusions. Three out of $24(4.2 \%)$ of the participants in the IL-4 condition emitted a total of 10 intrusions, and 9 out of $26(34.6 \%)$ participants in the IL-8 group produced 16 intrusions. Given that each participant had 10 
opportunities ( 1 per cue) to make an intrusion, intrusion ratios can be calculated. There is a slight tendency for intrusion ratios to increase over the three degree-of-IL conditions: IL- $1=.028$, IL-4 $=.041$, IL-8 $=.061$. In order to estimate how List 1 recall would appear if all intrusions were, in actuality, erroneous selections of List 2 targets in the face of source confusion when both targets were remembered, data were reanalyzed after intrusion scores had been added to the participants' recall scores. The analysis resulted in no substantive changes from the conclusions already reported.

The fact that all $\mathrm{AB}$ and $\mathrm{AC}$ pairs were person-location pairs could have contributed to undetected covert source memory confusion that resulted in an overestimation of recall decrements. Experiment 2 incorporated two procedures to reduce overt and covert source memory confusion. The list unit was an individual word, and recall was tested with stem recall cues. Stem recall not only should reduce source confusion, but also should provide a further guard against output interference by minimizing the participants' tendencies to covertly recall more recently studied items at the time of testing (cf. Anderson \& Bell, 2001).

The possibility that the participants engaged in increasing amounts of uncontrolled covert retrieval practice with increased exposure to the interpolated list was not necessarily ruled out by the nominal study-only conditions of interpolation. Given that learning was intentional and that the participants were tested for List 1 recall prior to exposure of List 2, it is not unlikely that the participants would rehearse List 2 in anticipation of a possible test of the material. Experiment 2 used incidental encoding of both lists with no recall test until after interpolation, to exert control over covert rehearsal (e.g., Dyne, Humphreys, Bain, \& Pike, 1990).

\section{EXPERIMENT 2}

Experimental participants viewed a list of 20 unrelated words under incidental learning instructions, after which they viewed another list either one or four times. The first two letters of each List 1 word were provided on the critical recall test.

\section{Method}

Participants and Materials. The participants were tested in groups. Students from intact laboratory sections or who volunteered for extra course credit were randomly assigned to conditions, with the restriction that there be 32 in each of the three conditions, one control (IL-0) and two experimental (IL-1 and IL-4). Two lists of 20 unrelated two-syllable nouns were selected from a local word pool such that no 2 out of the 40 began with the same first two letters. Each list served as the first list for half of the participants in each condition and as the second list for the other 16 participants in each experimental condition.

Procedure. Lists were presented on a TV monitor under incidental learning instructions. Booklets were provided for imagery ratings and for retention testing. The orienting task asked the participants to use a 5-point scale to rate how easy it was to form an image from the word. Each word was exposed for $4 \mathrm{sec}$, followed by a 6-sec blank screen for ratings. After the first list had been pre- sented for one cycle, the experimental participants were informed that a second set of words would be presented in the same way. The IL-4 participants were informed that they would see each word in the second set four times. The IL- 0 participants worked on a cartoonrating task for the amount of time required for four cycles of List 2 exposure.

After the participants had completed the second task, they received a 4-min stem cued recall test for first-list words. The instructions stressed that the participants were to try to remember words from the first set and that the test booklet contained 20 lines, each of which contained the first two letters of a first-list word. Next, a 4-min free recall test of both lists was administered, followed by a 7-item postexperiment questionnaire with two items designed to assess intentional learning ("I deliberately attempted to learn or memorize the words in the sets") and expectations of retention testing ("I expected that I would be given the opportunity to remember the words"). The participants were asked to use a 5-point scale $(1=$ definitely yes, $2=$ yes, $3=$ uncertain, $4=$ no, and $5=$ definitely no) to describe how much the items pertained to them. Four participants ( 1 each from IL- 0 and IL-4 and 2 from IL- 1 ) who answered definitely yes or yes to either of these items were replaced. ${ }^{1}$

\section{Results}

Mean List 1 scores (as percentages, with standard deviations in parentheses) on the word stem cued recall test following the interpolated task for the IL-0, IL-1, and IL-4 groups were $44.1 \%$ (21.27), 38.4\% (14.28), and 32.5\% (12.83), respectively. The variation among these means was statistically significant $\left[F\left(2,93=3.91, M S_{\mathrm{e}}=\right.\right.$ 273.59, $p<.001]$, and they are presented in Figure 1 . The Bonferroni method $(\alpha=.05)$ was used to adjust for multiple comparisons. A single exposure to the IL was not sufficient to produce RI, as is indicated by the nonsignificant IL-0 versus IL-1 comparison. However, the significant IL-0 versus IL-4 comparison reveals that four cycles of the IL yielded reliable RI. The difference between the IL-1 and the IL-4 conditions was not significant. Comparison of List 2 percentages of free recall (standard deviations in parentheses) [IL-1 $=23.4 \%$ (12.1); IL-4 $=61.6 \%$ (14.0)] indicates that incidental learning of the second list increased considerably with additional exposures $[t(62)=11.67, p<.001]$.

\section{GENERAL DISCUSSION AND CONCLUSIONS}

Until recently, we did not question that RI was directly linked, in one way or another, to new associative learning. Certainly, interpolated learning seems to be a critical condition for response decrements of the RI class. However, until Anderson (2003; Anderson et al., 1994) identified the confound between interpolated learning and retrieval practice during most interpolated learning and the theoretical implications of this, there was no compelling reason to experimentally dissociate new learning from retrieval practice. Anderson's insight and associated research have contributed to a reinvigorated interference construct in cognitive psychology. It is possible that RI results only from inhibition that develops from the participant's active attempts to cope with interfering competing responses, not from new learning per se, and 
that RI will not occur if interfering material is learned under conditions that do not give rise to interferenceactivated inhibitory mechanisms (Anderson, 2003). Although a large body of research supports retrieval-induced inhibition as an underlying mechanism of response decrements in preparations related to those of conventional RI experiments, Bäuml (1996) appears to be the only extant attempt to determine whether RI is eliminated when retrieval-induced inhibition is unlikely. As was pointed out above, Bäuml obtained a considerable amount of RI when experimental conditions were compared with (a less than ideal) control condition.

Taken together, the present two experiments present a reasonably convincing case that all RI is not the result of retrieval-induced inhibition. RI was obtained with intentional learning of $\mathrm{AB}-\mathrm{AC}$ pairs and no overt retrieval practice in Experiment 1. Experiment 2 yielded RI with conditions that differed considerably from those in the first experiment. The conditions in Experiment 2 both extended the domain of testing for RI in the absence of retrieval practice and addressed possible alternative interpretations of the outcome of the first experiment. Both experiments produced a retention curve in which amount of RI was a negatively accelerated increasing function of degree of interpolated learning. In neither experiment did RI reach asymptote at the lowest level of IL.

The results reported here do not suggest that retrievalinduced inhibition is not a factor in RI. They do suggest that not all RI is attributable to inhibitory processes induced by actual or functional retrieval practice. One can envision the exciting possibility of fruitful research on forgetting that incorporates techniques designed to tease apart truly inhibitory (per Anderson, 2003) and other underlying mechanisms.

\section{REFERENCES}

Anderson, M. C. (2003). Rethinking interference theory: Executive control and the mechanisms of forgetting. Journal of Memory \& Language, 49, 415-445.

ANDERSON, M. C., \& BeLL, T. (2001). Forgetting our facts: The role of inhibitory processes in the loss of propositional knowledge. Journal of Experimental Psychology: General, 130, 544-570.

Anderson, M. C., BJorK, E. L., \& BJorK, R. A. (2000). Retrievalinduced forgetting: Evidence for a recall-specific mechanism. Psychonomic Bulletin \& Review, 7, 522-530.

ANDERSON, M. C., BJORK, R. A., \& BJORK, E. L. (1994). Remembering can cause forgetting: Retrieval dynamics in long-term memory. Journal of Experimental Psychology: Learning, Memory, \& Cognition, 20, 1063-1087.

BARNES, J. M., \& UNDERWOOD, B. J. (1959). "Fate" of first-list associations in transfer theory. Journal of Experimental Psychology, 58, 97-105.

B ̈̈UML, K.-H. (1996). Revisiting an old issue: Retroactive interference as a function of the degree of original and interpolated learning. Psychonomic Bulletin \& Review, 3, 380-384.

BRIGGS, G. E. (1957). Retroactive inhibition as a function of degree of original and interpolated learning. Journal of Experimental Psychology, 53, 60-67.
Chappell, M., \& Humphreys, M. S. (1994). An auto-associative neural network for sparse representations: Analysis and application to models of recognition and cued recall. Psychological Review, 101, 103128.

Dyne, A. M., Humphreys, M. S., Bain, J. D., \& Pike, R. (1990). Associative interference effects in recognition and recall. Journal of Experimental Psychology: Learning, Memory, \& Cognition, 16, 813824.

LEWANDOWSKY, S. (1991). Gradual unlearning and catastrophic interference: A comparison of distributed architectures. In W. E. Hockley \& S. Lewandowsky (Eds.), Relating theory and data: Essays on human memory in honor of Bennet B. Murdock (pp. 445-476). Hillsdale, NJ: Erlbaum.

McCloskey, M., \& Cohen, N. J. (1989). Catastrophic interference in connectionist networks: The sequential learning problem. In G. H. Bower (Ed.), The psychology of learning and motivation (Vol. 24, pp. 109-165). San Diego: Academic Press.

McGeoch, J. A. (1932). The influence of degree of interpolated learning upon retroactive inhibition. American Journal of Psychology, 44, 695-708.

Melton, A. W., \& Irwin, J. M. (1940). The influence of degree of interpolated learning on retroactive inhibition and the overt transfer of specific responses. American Journal of Psychology, 53, 173-203.

Mensink, G. J., \& RaAiJmakers, J. G. W. (1988). A model for interference and forgetting. Psychological Review, 95, 434-455.

Postman, L. (1971). Transfer, interference and forgetting. In J. W. Kling \& L. A. Riggs (Eds.), Woodworth and Schlosberg's Experimental psychology (3rd ed., pp. 1019-1132). New York: Holt, Rinehart \& Winston.

Postman, L. (1976). Interference theory revisited. In J. Brown (Ed.), Recall and recognition (pp. 157-181). New York: Wiley.

Postman, L., \& KePPel, G. (1967). Retroactive inhibition in free recall. Journal of Experimental Psychology, 74, 203-211.

Postman, L., \& Riley, D. A. (1959). Degree of learning and interserial interference in retention: A review of the literature and an experimental analysis. University of California Publications in Psychology, 8, 271-396.

Shadish, W. R., CooK, T. D., \& CAmpbell, D. T. (2002). Experimental and quasi-experimental designs for generalized causal inference. Boston: Houghton Mifflin.

SLAMECKA, N. J. (1960). Retroactive inhibition of connected discourse as a function of practice level. Journal of Experimental Psychology, 59, 104-108.

SMITH, A. D. (1971). Output interference and organized recall from long-term memory. Journal of Verbal Learning \& Verbal Behavior, 10, 400-408.

UNDERWOOD, B. J. (1983). Attributes of memory. Glenview, IL: Scott, Foresman.

UNDERwOOD, B. J., \& LUND, A. M. (1981). The effect of degree of learning on within-list competition. American Journal of Psychology, 94, 195-207.

\section{NOTE}

1. The means for retained participants (with standard deviations in parentheses) in each of the three conditions (IL-0, IL-1, and IL-4) for the "attempted to learn" item were $4.50(0.62), 4.41(0.56)$, and 4.47 $(0.67)$, respectively $\left[F(2,93)=0.19, M S_{\mathrm{e}}=0.38, p=.83\right]$. Comparable values for the "opportunity to remember" item were $3.88(0.71)$, $4.00(0.76)$, and $4.22(0.83)$ for the IL-0, IL-1, and IL-4 conditions, respectively $\left[F(2,93)=1.64, M S_{\mathrm{e}}=0.59, p=.20\right]$. As would be expected on the basis of the statistically nonsignificant variation among these sets of means, when scores on these two ratings were included as covariates in analyses of List 1 recall, the conclusions did not depart from those reported in the Results section.

(Manuscript received April 2, 2003; revision accepted for publication May 25, 2004.) 